\title{
Protective Immunity Against Cryptococcus Neoformans Infection
}

\author{
Joel Schop*
}

\begin{abstract}
Cryptococcus neoformans, the etiological agent of cryptococcosis, is an occasional opportunistic fungal pathogen of immune competent individuals. However, it is a relatively frequent cause of life-threatening meningoencephalitis and pulmonary infections in immunosuppressed hosts and is a leading mycological cause of morbidity and mortality among patients with AIDS in most parts of the world. The lack of an effective fungicidal regimen and the development of antifungal resistant strains suggest that continued investigation is necessary to devise immunotherapeutic strategies, drug targets and/or vaccines to combat $C$. neoformans infections. Until recently, cryptococcal virulence factors such as its polysaccharide capsule, macrophage parasitism, and its ability to induce an ineffective antibody mediated immune (AMI) response along with a non-protective type II (Th2) cellmediated immune response have thwarted efforts to induce complete protective immunity against a lethal cryptococcal strain in murine models. The presence of $\mathrm{C}$. neoformans antibodies in adult human serum suggests that immune competent individuals have difficulty resolving an early cryptococcal infection allowing for the establishment of a subclinical chronic infection. Recent studies have shown that pro-inflammatory cytokines, specifically interferon-g (IFN- $\gamma$ ), associated with type I (Th1) cellmediated immunity can successfully drive cell-mediated immune (CMI) responses to produce protective immunity to a second experimental $C$. neoformans infection in mice. This review will evaluate the intricacies of the host-cryptococcal interaction and discuss recent developments in $C$. neoformans research and the potential for human vaccines and/or drug therapies.
\end{abstract}

\section{INTRODUCTION}

Cryptococcus neoformans, the causative agent of cryptococcosis, is a common encapsulated fungus that can cause a range of illnesses from arthritis to prosthetic valve implant infections and, most significantly, a lethal infection of the central nervous system (CNS) leading to meningoencephalitis, predominantly in immunocompromised individuals $(1,2)$. With the eruption of the HIV epidemic, C. neoformans has only emerged as a serious human pathogen in the last 30 years and has become the leading mycological cause of morbidity and mortality among AIDS patients $(3,4)$. It

\footnotetext{
* To whom correspondence should be addressed: Joel Schop 4041 Medical Dr. Apt 1718, San Antonio, Tx 78229

Tel: (210) 373-8210j

E-mail: schop@lonestar.utsa.edu
}

is estimated that $6 \%$ to $10 \%$ of patients with AIDS in the United States, Western Europe, and Australia and $0 \%$ to $50 \%$ of AIDS patients in sub-Saharan Africa countries are infected with life-threatening cryptococcal meningitis $(5,6)$. By the 1990s, C. neoformans had become the leading cause of culture-positive meningitis in many regions including New York City (7).

Although this review will focus on cryptococcosis which predominantly occurs in immunocompromised individuals, increasing reports of CNS cryptococcosis of immunocompetent, HIV-negative patients have been described (8, 9). In a recent study, Ecevit et al. evaluated the poor prognosis of 9 nonimmunosuppressed patients with CNS cryptococcosis. Seventy-seven percent of the individuals had no underlying medical conditions and all 9 patients were eventually treated with amphotericin B/azole therapy. 
The reported mortality rate was $44 \%$ while the rest suffered neurological sequelae (9).

With amphotericin B/azole therapy and the advent of highly active anti-retroviral therapy (HAART), incidences of cryptococcosis have declined in HIVinfected patients in the developed parts of the world (10). However, the increase in HIV-negative, immunocompetent patients receiving immunosuppressive therapy, the large numbers of people without economic access to HAART and the high mortality and morbidity of HAART-related immune reconstitution inflammatory syndrome (IRIS) (11) begs the necessity for the development of a $C$. neoformans vaccine. This review will discuss the recent progress in the understanding of cryptococcal-host interactions and the challenges involved with the development of protective immunity against $C$. neoformans.

\section{THE FUNGUS AND VIRULENCE FACTORS}

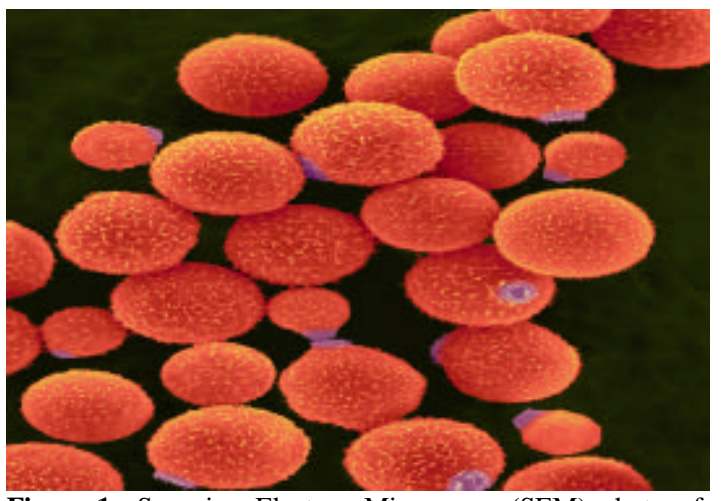

Figure 1. Scanning Electron Microscope (SEM) photo of encapsulated pathogenic Cryptococcus neoformans yeast at a magnification of $\mathrm{x} 1,200$. An acidic mucopolysaccharide capsule completely encloses the fungus. C. neoformans infection, cryptococcosis may lead to meningoencephalitis of the central nervous system (CNS) predominantly in immunocompromised humans with HIV/AIDS or undergoing immunosuppressive therapy. Copyright Dennis Kunkel Microscopy, Inc.

C. neoformans is usually found in yeast form, or oval or spherical shape (Figure 1). Generally, the yeast form reproduces by asexual budding, but sexual reproduction has been observed with the formation of basidiospores sexual spores produced at the end of hyphae by members of the phylum Basidiomycota of which Filobasidiella neoformans (the sexual state of $C$. neoformans) is a member. Until recently, $C$. neoformans has been subdivided into two variants (var.), $C$. neoformans var. neoformans and $C$. neoformans var. gattii. However, a greater analysis of the genetic structure of $C$. neoformans strains has resulted in the elevation of $C$. neoformans var. gattii to species level (12). Cryptococcal isolates are also categorized according to serotype based upon antigenic differences in their polysaccharide capsules. Serotypes A, D, and hybrid $\mathrm{AD}$ belong to $C$. neoformans and serotypes $\mathrm{B}$ and $\mathrm{C}$ belong to $C$. gattii. $C$. neoformans serotype A appears to be implicated in $99 \%$ of AIDS patients with cryptococcosis worldwide, except France where serotype $\mathrm{A}$ is responsible for around $80 \%$ of the infections (13). More frequent cases of serotype D and $\mathrm{AD}$ have been reported in Europe where cryptococcosis is associated with $77 \%$ of HIV patients (14). Serotype A contributes to $51 \%$ of $C$. neoformans infection followed by serotype D (30\%) and serotype AD (19\%) (14). Given that $C$. neoformans serotype A still remains to be the most prevalent variety amongst immunocompromised individuals, we will confine the majority of our discussion of cryptococcal immunity and virulence characteristics to Cryptococcus.

C. neoformans Serotype A incorporates a multitude of virulence factors to overcome host defenses, including the ability to produce a variety of anti-oxidants. Melanin is a free-radical scavenger that impedes macrophage phagocytosis by helping to protect $C$. neoformans against nitrogen- and oxygen-derived oxidants produced as defense mechanisms by the host $(15,16,17)$. Superoxide dismutase supplements melanin by converting superoxide radicals into hydrogen peroxide and molecular oxygen $(18,19)$. Thioredoxin reductase and mannitol are also powerful anti-oxidants produced by this fungal pathogen $(20,21)$.

Besides its resistance to oxidative stress, $C$. neoformans owes much of its uniqueness and pathogenic virulence to its polysaccharide capsule. Not only does the capsule provide a protective barrier around the fungal cell wall but it also contains particular capsular antigens, such as glucuronoxylomannan (GXM), that have been suspected to elicit an adverse immune response, allowing the microbe to escape significant host phagocytosis and intercellular killing $(22,23,24)$.

Despite its virulence, a $C$. neoformans infection is usually contained in immunocompetent hosts. Sera studies suggest that the majority of the human population is initially infected during early childhood and repeatedly infected throughout life $(25,26)$. Because of the rarity of clinical manifestation of cryptococcosis in normal individuals, we can assume that the host mounts an immune response that may not completely eliminate the infection, but successfully prevents disease. Therefore, a clinical cryptococcal infection in humans, later in life, would more than likely result from reactivation of a latent infection or an acute re-infection in the contexts of an established chronic infection.

\section{HOST DEFENSE}

Route of infection and innate immunity 
Although often overlooked, it is important to note certain physical factors and barriers that impede the establishment of $C$. neoformans in the mammalian environment. The initial defense to all fungal infections is the skin. Since the skin provides an effective barrier to $C$. neoformans, the nasal and upper respiratory airway openings appear to be critical host entry sites. Kuttin et al. (27) have established that $C$. neoformans has the ability to cross the mucosal and nasal epithelial layers in mice and rats. The connection between the nasal cavity and subcranial space suggests a possible $C$. neoformans entry route into the central nervous system (CNS). The degree to which this mode of passage facilitates infection has yet to be defined.

It is commonly believed that inhalation is the primary route of $C$. neoformans pulmonary infection in humans. Considering C. neoformans grows less efficiently at the human host temperature of $37^{\circ} \mathrm{C}$ than at its optimal growth rate temperature of $25^{\circ} \mathrm{C}$ to $30^{\circ} \mathrm{C}$ (28), low temperatures in the nasal passage might be advantageous for fungal growth.

Ciliary action and airway turbulence are generally successful at preventing yeast cells from reaching the alveoli, except the smaller basidiospores. Invasion of the bronchial epithelium is sufficient to inflict direct host damage and/or trigger an over-reactive inflammatory response (29). For instance, $C$. neoformans var. gatti has been associated with the vigorous formation of granulomatous masses in the lungs (30). A more severe systemic infection, mainly seen in immunocompromised patients, can lead to $C$. neoformans meningoencephalitis - a symptomatic infection that generally becomes fatal if not promptly treated.

\section{COMPLEMENT-MEDIATED ANDNON - SPECIFIC CELLULAR IMMUNITY}

The complement system consists of a series of serum proteins that are involved in non-specific host immune responses. Complement proteins can be rapidly activated to provide protection to the host via multiple mechanisms including opsonization which promotes phagocytosis of antigens, the lysis of foreign cells, and the activation of inflammatory responses. Complement system activation can occur via three pathways: complement activation via the classical pathway is initiated by the formation of antigen-antibody complexes and requires specific antibody; activation of the alternative pathway begins with binding of serum proteins including the complement protein $\mathrm{C} 3 \mathrm{~b}$ to the foreign cell surface and does not require the presence of antibody; lastly, the lectin pathway requires the binding of lectins (proteins that bind specifically to carbohydrate targets) to mannose residues on foreign cells and, like the alternative pathway, does not require specific antibodies.

The level of complement cascade activation by $C$. neoformans surface antigens depends on the integrity of its capsule and the presence of particular antifungal antibodies in the host serum at the time of infection (31). Although human serum contains sufficient antibodies for $C$. neoformans glucans, the polysaccharide capsule blocks access to the yeast surface (32). In naive host sera, there are insufficient naturally occurring capsule-binding antibodies to initiate the classical pathway (33). Therefore, the alternative pathway remains the primary method for activation of complement mediated phagocytosis of encapsulated $C$. neoformans. Immunofluorescence studies have shown that the classical pathway results in the immediate accumulation of C3 (a key complement protein) on the cell surface, while the alternative pathway activation lags behind 4 to $7 \mathrm{~min}$ before significant $\mathrm{C} 3$ is present $(33,34)$. These findings propose that the polysaccharide capsule is a key factor in the ability of $C$. neoformans to slow the humoral complement-mediated immune response.

Phagocytosis appears to be the primary method for clearing C. neoformans infection. Various host defense cells, employing a multitude of microbicidial mechanisms, contribute to this process. Polymorphonuclear (PMN) cells, also known as neutrophils, are effective phagocytic cells in the early stages of infection and may serve the immune system best by containing a chronic $C$. neoformans infection (35). In a recent study, Marr K. et al. (36) explored the similarities and differences of natural killer (NK) cells anticryptococcal activity between humans and mice. NK cells can play different roles in humans than in mice during cryptococcal infection and vary in respect to their distribution in the body, cytokine interaction, receptor expression and their interaction with a variety of other effecter molecules $(36,37)$. However, macrophages undoubtedly play an essential role in $C$. neoformans phagocytosis throughout the duration of the infection in humans and mice. Macrophages are professional phagocytic cells capable of ingesting foreign cells via contact with antibody $F c$ receptors and/or C3 receptors by means of the complement pathways. Due to human host inefficiency to produce a consistent amount of protective antibodies or the inability to upregulate the necessary cytokines to elicit an effective cell-mediated immune response, macrophage phagocytosis mainly occurs via the alternative complement pathway (34).

During the immune response, macrophages and human PMN cells produce an assortment of reactive oxygen intermediates (ROIs) and nonoxidative mechanisms that are capable of killing $C$. neoformans $(38,39,40)$. Human neutrophils produce hydrogen 
peroxide $\left(\mathrm{H}_{2} \mathrm{O}_{2}\right)$ which, in adequate amount is fungicidal to C. neoformans (39). Phagocytes also produce non-oxidative antimicrobial proteins. Human neutrophils contain defensins which can be extremely lethal to $C$. neoformans fungus (38). Murine macrophages produce a histone-like microbicidial protein which is also an effective cryptococcal killing mechanism (40).

Although human phagocytes produce effective antifungal molecules, many studies have shown that an adequate cryptococcal defense involves macrophages with sufficient stimulus to successfully phagocytosis and kill C. neoformans (41). Flesh et al. (42) demonstrated that murine (mouse) bone marrow macrophages, in the presence of IFN-gamma and bacterial lipopolysaccharide (LPS), can mount an antifungal attack against $C$. neoformans in the absence of complement and antibody opsonins. It has also been shown that cytokines, specifically granulocytemacrophage colony-stimulating factors (GM-CSF) and tumor necrosis factor-alpha (TNF-a) enhance complement-mediated phagocytosis in vitro and in murine models (43). Without these cytokines, macrophages only ingested 1 to 2 cryptococcal cells as compared to 6 to 8 with appropriate cytokine stimulation (43). Although a macrophage is fully capable of $C$. neoformans phagocytosis, this does not necessary imply death of the fungus. $C$. neoformans has demonstrated the ability to survive and replicate within macrophages in vitro and in vivo $(44,45,46)$.

In summary, macrophages are proficient killers of $C$. neoformans by phagocytosis, but are greatly dependent on the presence of serum opsonins and cytokinemediated activation. The polysaccharide capsule provides an effective barrier against complementmediated opsonization. Evidence points towards the appropriate immune cytokine response as being a key factor in the ability of macrophages to effectively clear a C. neoformans infection.

\section{SPECIFIC IMMUNITY Antibody-mediated immunity}

A correctly implemented immune response that incorporates phagocytic killing mechanisms and the appropriate memory T-cell and B-cell response is required to mount an adequate immune defense against C. neoformans. Fortunately, extensive study has been conducted on antibody production to cryptococcal polysaccharide antigens. However, not all $C$. neoformans antigens elicit protective antibody production. The efficiency of an antibody-mediated response is determined by monoclonal antibody $(\mathrm{mAb})$ isotype (having to do with $\mathrm{Fc}$ region: $\operatorname{IgG}, \operatorname{IgM}, \operatorname{IgA}$, $\operatorname{IgE}, \operatorname{IgD}$ ), epitope specificity (immunoglobulin hypervariable region specificity to $C$. neoformans antigens) and affinity for different $C$. neoformans strains. The antibody-mediated immunity (AMI) and the cellmediated immunity (CMI) responses to capsular glucuronoxylomannan (GXM) sugars and cryptococcal mannoproteins are the subject of the majority of cryptococcal antigen studies to date.

Antibodies to $C$. neoformans polysaccharide capsule are commonly found in immunocompetent individuals with or without cryptococcal infection (26). Perhaps, this antibody response is due to a past subclinical infection (no manifestation of characteristic symptoms of a $C$. neoformans infection) or a cross reaction with other polysaccharides. Whatever the case, it is apparent that immunocompetent individuals have some degree of immunity to $C$. neoformans polysaccharide antigens.

GXM capsular sugars by themselves elicit very little antibody immune responses and can wreak havoc on the host immune system (47). However, when linked to a protein carrier, such as tetanus toxoid (TT) or Pseudomonas aeruginosa exoprotein A (rEPA), it becomes highly immunogenic (48, 49). GXM conjugated to tetanus toxoid (GXM-TT) has been successful at providing partial protective antibody production in only some mouse species (48). Not only does this suggest that a GXM-TT protective immune response depends on the host's genetic framework, but studies have shown that GXM-TT was unsuccessful at inducing partial protective immunity in mice with $\mathrm{T}$ cell, IFN- $\gamma$, iNOS, B cells, and various other CMI cytokine deficiencies (50). This is a significant drawback considering that the majority of human cryptococcal cases are those with HIV or immunocompromised patients with low T-cell levels. GXM-TT vaccine was proven to be immunogenic in immunocompetent humans in a small clinical trial, but its immunogenicity in immunocompromised individual is still unclear (51).

This evidence suggests that a successful protective AMI is greatly dependent on functional CMI and the appropriate cytokine immune response. In the field of cryptococcal research, it has generally been accepted that $C$. neoformans capsular polysaccharides induce Tcell-independent antibodies and, therefore, elicits a Th2 immune response. In the above mentioned experiment, it seems that the inactivation of T-cells and the lack of Th1 cytokines, such as IFN- $\gamma$, IL-12, and TNF-a, may have interfered with the production of "protective" antibodies.

Furthermore, disease-enhancing antibodies have been described. The presence of too many antibodies of the wrong type has been observed to produce "immunological paralysis" or antibody unresponsiveness $(22,51)$. Mice injected with more than 100-400 $\mu \mathrm{g}$ of cryptococcal polysaccharide demonstrated a reduced ability to produce an antibody 
response as compared to mice injected with less than $100 \mu \mathrm{g}(22)$. Apparently, the production of antibodies to particular $C$. neoformans capsular antigens can "overload" the immune system. The effects of this phenomena and the degree of T-cell involvement in this response have not been thoroughly investigated in humans.

In summary, AMI to C. neoformans appears to be complex. Convincing evidence supports the interdependency of CMI and AMI in a successful host defense response against $C$. neoformans. Considering that immunocompetent individuals are capable of producing only minimally protective antibodies to the polysaccharide, the cooperation of the CMI and the correct T-helper cell type cytokine response might be a pivotal step to induce antibody-mediated protection. The immunocompromised host's inability to mount an effective AMI attack against $C$. neoformans infection because of deficient or inappropriate $\mathrm{T}$ cell activation also points to the importance of anti-cryptococcal CMI responses.

\section{Cell-Mediated Immunity}

The foremost reasons to suspect significant T-cell involvement in host immune response to $C$. neoformans infection are 1) the difficultly of CMI deficient patients to successfully mount an anti-cryptococcal immune response, 2) the formation of granulomas usually associated with extensive Th1 activation, 3) the experimental evidence that naïve mice can adopt protective immunity by $\mathrm{T}$-cell transfer from immune mice, and 4) successful stimulation of complete acquired protective immunity in mice inoculated with a C. neoformans strain producing host IFN- $\gamma(3,4,30,52$, 53). Besides the fact that the T-helper cell plays the central role in the specific immune response, as well as the humoral response, and is essential for any successful cryptococcal defense, many experiments provide conclusive evidence that CMI is vital to the development of protective immunity against $C$. neoformans.

Experiments have been performed with athymic mice $(\mathrm{nu} / \mathrm{nu})$ that cannot efficiently facilitate a T-cell mediated immune response. These mice were more susceptible to the $C$. neoformans infection compared to the control mice with a thymus (54). Moreover, athymic mice were shown to be susceptible to chronic cryptococcal infection when presented with an avirulent nonencapsulated $C$. neoformans strain (55). Upon examination, nu/nu mice presented no granuloma formation, less inflammatory evidence, and negative delayed type hypersensitivity (DTH) responses $(54,56)$. Similar studies of pulmonary infections in rats show that $C$. neoformans in the lungs of nu/nu rats are lethal (57). Normal rats can effectively clear a C. neoformans pulmonary infection. This evidence suggests that athymic mice and rats are unable to ward off a $C$. neoformans infection due to the lack of mature T-cells in their serum.

Further studies implementing adoptive transfer technique of mouse splenocytes have been performed to analyze the potential for T-cell protective immunity (52). After 35 days of cryptococcal infection, splenic Tcells transferred to naïve mice produced a DTH reaction and protective immunity when presented with $C$. neoformans (52). However, transfer of infected serum did not confer significant immunity to the naïve host (58). These studies seem to suggest that protective immunity is possible in mice and is associated with Tcells.

Wormley et al. $(53,58)$ have conducted 2 studies analyzing the protective immune response to $C$. neoformans by way of Th1 cytokine responses in mice. Over a period of 14 days, cytokine responses, CD4+ and CD8+ cell levels and pulmonary fungal burden were recorded in mice challenged with known lethal $C$. neoformans strain $\mathrm{H} 99$ and an avirulent temperaturesensitive, calcineurin A1 (cnal) mutant strain (59).

Cnal strains have been engineered to inefficiently propagate a virulent infection in simulated host environments $\left(37^{\circ} \mathrm{C}, 5 \% \mathrm{CO} 2\right.$, etc. $)$ and have been shown to be non-infectious in immunosuppressed murine model studies (59). The mice presented with the cnal strains successfully resolved the infection while mice challenged with the virulent strain all died within a median of 16 days (58). However, the cnal mice did not demonstrate protection against a re-challenge with pathogenic $C$. neoformans strain H99 (58). When inoculated with a second lethal $C$. neoformans strain, the cnal mice only survived approximately 17 days almost the same survival interval as the control group inoculated with lethal C. neoformans H99 strain (58). During the study, IL-4 levels increased in mice inoculated with $10^{6} \mathrm{CFU}$ of cnal mutant, indicative of a Th2 response (58). In addition, IFN-gamma and IL-2 (Th1) cytokines levels did not significantly deviate from the control norms in the cnal mice as compared to a serious, steady decline in mice inoculated with the lethal strain (58). Considering the multitude of studies showing the importance IFN- $\gamma$ in the development of protective CMI to $C$. neoformans, there is significant evidence to conclude that cnal inoculated mice failed to produce substantial amounts of INF-gamma or initiate a significant Th1 response to procure protection against a secondary infection.

Knock-out (KO) studies by Hernandez et al. (60) have also confirmed that a Th2 driven response exacerbates $C$. neoformans infection. IL-4 KO and LI$10 \mathrm{KO}$ (cytokines affiliated with a Th2 response) mice 
both resolved $C$. neoformans infection at a significantly higher rate than wildtype C57BL/6 mice (60). In contrast, treatment of cryptococcal resistant mice with IL-4 and IL-10 increased fungal presence in the CNS (61).

In light of these conclusions, follow-up studies by Wormley et al. (53) focused on the activation of a Th1 response in murine models. They were able to transform C. neoformans strain $\mathrm{H} 99$ with a construct allowing the yeast to express low levels of the Th1-type cytokine IFN- $\gamma$. The strain was designated $C$. neoformans strain H99 (53). Besides its production of IFN-gamma, this strain showed no signs of attenuated virulence when compared to the known lethal, wildtype $C$. neoformans strain $\mathrm{H} 99$ in vitro (53). When $\mathrm{A} / \mathrm{Jcr}$ mice were given intranasal inoculations of C. neoformans strain $\mathrm{H} 99 \gamma$, they were able to resolve a primary infection compared to complete mortality for mice inoculated with the wildtype strain (53). Importantly, A/Jcr mice are deficient in the complement protein $\mathrm{C} 5 \mathrm{a}$, a protein that is significant in the recruitment of inflammatory cells during inflammatory responses. The capacity of prior infection with the IFN- $\gamma$-producing strain to induce protective anti-cryptococcal immunity in an "immune deficient" mouse strain speaks very highly of the level of protection that this strain generated against $C$. neoformans. To evaluate the possibility for acquired protective immunity, immunocompetent $\mathrm{BALB} / \mathrm{c}$ mice that had resolved a previous primary infection with $C$. neoformans strain $\mathrm{H} 99 \gamma$ and mice that had survived a primary infection with heat-killed $C$. neoformans strain were both inoculated with a secondary infection of the lethal wildtype C. neoformans strain H99. All mice that had resolved a prior infection with the $C$. neoformans strain $\mathrm{H} 99 \gamma$ were able to survive a second cryptococcal challenge with the pathogenic wild-type strain demonstrating the development of complete protective immunity as compared to $100 \%$ mortality of the mice that had resolved the prior infection with heat-killed $C$. neoformans (53).

Cytokine and chemokine analysis revealed that pulmonary levels of Th1 cytokines, IFN- $\gamma$, IL-2, IL-12, and TNF-a were substantially elevated as well as other inflammatory cytokines and chemokines in H99 $\gamma$ mice (53). In contrast, the above mentioned cytokines and chemokines levels were significantly lower in the wildtype infected mice (53). However, wild-type infected mice did show increased levels of Th2 cytokines, IL-4 and IL-5 as compared to the C. neoformans strain H99y immunized mice (53). These results suggest that complete protective immunity is possible in mice when a Th1 cytokine response is induced upon primary infection with C. neoformans $\mathrm{H} 99 \mathrm{y}$ strain.

In summary, $100 \%$ survival of a primary infection of the $C$. neoformans strain producing IFN- $\gamma$ and the activation of protective immunity in mice is promising. Experiments show that successful cryptococcal protection is strongly connected to CMI, particularly the activation of a Th1 response and Th1 cytokines, such as IFN- $\gamma$, IL-12, and TNF-a. Results showing that susceptible mice naturally produce $\mathrm{Th} 2$ cytokines, with a diminished Th1 response, when inoculated with a pathogenic cryptococcal strain suggests that $C$. neoformans escapes destruction by mediating a biased Th2 immune response $(53,58)$. It is possible to propose that the human immune system suffers from the same debilitating response to $C$. neoformans and without elevated Th1 cytokines, the AMI and Th2 response can only produce partial protective immunity. Although this response might be adequate in the immunocompetent host, if this balance is tipped, as it is in patients suffering from AIDS, the host defense against $C$. neoformans invasion could be severely impaired. The process by which Th1 cytokine polarization stimulates anti-cryptococcal protective immunity remains to be thoroughly investigated.

\section{MANNOPROTEINS}

Host immune responses to cryptococcal proteins have been associated with protection against experimental infection in mice. Vaccination of mice with a $C$. neoformans culture filtrate antigen $(\mathrm{CneF})$ in complete Freund's adjuvant (CFA) has been shown to induce partial-protection against a subsequent cryptococcal infection as well as to generate DTH responses (62). Fractionation of CneF revealed its mannoproteins (MP) fraction as the primary antigenic component responsible for the stimulation of anti-cryptococcal CMI responses in mice (62). Subsequently, there has been great interest in identifying the proteins that elicit protective host responses to cryptococcal infection. Mandel et al. (63) have identified and cloned a gene, designated DHAl, which encodes a protein that induces DTH responses in mice. Additionally, Biondo et al. (64) have described a cryptococcal polysaccharide deacetylase that was used as a vaccine strategy to prolong survival and decrease fungal burden in mice. Levitz et al. (65) have also described a $C$. neoformans mannoprotein, termed MP98, which has molecular properties of a chitin deacetylase and stimulates $\mathrm{T}$ cell responses to $C$. neoformans. A second cryptococcal mannoprotein, MP88, also identified by Levitz and collaborators was also demonstrated to stimulate $\mathrm{T}$ cell responses (66). More recently, two cryptococcal mannoproteins, designated MP84 and MP115, were identified following the reaction of various $\mathrm{CneF}$ mannoprotein fractions with sera from patients and experimental animals with cryptococcosis (67).

Current research suggests that mannoproteins activate a Th1 protective anticryptococcal response by 
presenting extensive o-mannosylated antigen to macrophage mannose receptors (MMR) on the surface of PMN cells, particularly dendritic cells $(68,69)$. The antigen is endosytosed and released in to the endosome to be processed for presentation on the major histocompatibility complex class II (MHC class II) (68). These antigen-presenting cells subsequently secrete IL12, generating Th1 cells and facilitating a proinflammatory response with elevated IFN-? production (70). In vitro research involving human monocytes and in vivo murine studies have demonstrated that IL-12 plays an essential role in the development of a protective immune response $(70,71)$. Pietrella et al. (72) also describe the stimulation of an IFN- $\gamma$ and IL-12-directed Th1 protective response in Candida albicans with cross-reactive MP from $C$. neoformans in mice and in vitro.

In summary, MPs, located in the C. neoformans envelope, are highly immunogenic and elicit DTH responses along with a partially protective immune response in mice $(63,71,73)$. T-cell involvement and increased secretion of IFN- $\gamma$, TNF-a, IL-2 (75) and IL12 (70) have been associated with particular mannoproteic determinants: MP98, MP88, MP84 and MP115 (65, 66, 67). While it has been demonstrated that purified capsular polysaccharides of $C$. neoformans promotes IL-10 secretion, among other Th2 cytokines that can downregulate proinflammatory Th1 cytokines (74), MPs have been shown to shift the balance toward a Th1 protective response. Although the value of these cryptococcal proteins as vaccine candidates for the prevention and/or treatment of C. neoformans infections or relapses in immunosuppressed patients have yet to be validated on a definitive basis, it appears that they have the potential to be of benefit for the management of cryptococcosis.

\section{VACCINE STRATEGIES}

As investigators get closer to discovering the intricacies of the host immune response to $C$. neoformans infection, the possibilities of a vaccine become more of a reality. It is promising to hear evidence of cross-reactivity in Th1 responses to $C$. neoformans and the pathogenic fungus, Candida albicans (72). These conclusions imply the potential for the first broad-spectrum vaccine that protects against various pathogenic fungi.

Development of a $C$. neoformans strain capable of producing host IFN- $\gamma$ is clearly a potential vaccine strategy, but the form, method and efficiency of this prospect in humans still needs to be investigated. Furthermore, host defense to C. neoformans is complex and probably entails an intricate collaboration of CMI, AMI and innate immunity. A better understanding of the Th1 response, how it can elicit protective immunity and the role that AMI plays in this process must be devised before a successful human vaccine candidate that induces complete protective immunity can be developed.

In addition to unanswered questions regarding anticryptococcal host defense mechanisms, an incorrectly polarized or severely impaired immune system is of essential interest when discussing cryptococcal vaccine strategies. Considering the majority of the $C$. neoformans clinical cases occur in immunocompromised patients (5), the potential of inciting an over-exuberant inflammatory response (immune reconstitution) during latent $C$. neoformans infection is a concern. This concern is apparent when examining the host immune system's ability to selfinflict damage during immune reconstitution inflammatory syndrome (IRIS) in HIV patients treated with HAART (11). Although studies with H99- $\gamma$ have been shown to ramp up Th1 cells, which might suggest a good strategy for AIDS and other immunocompromised patients, it is unknown if such methods will be successful in these populations.

\section{CONCLUSION}

With its polysaccharide capsule and its ability to evade the bulk of immune defenses by driving a nonprotective Th2 response, C. neoformans has proven to be one of the most challenging fungal infections of the $21^{\text {st }}$ century. Microbiologists and immunologists continue to decipher the cryptococcal code in order to better understand its mechanisms of virulence. An AMI response with antibody production from cryptococcal antigen has only been demonstrated to elicit partial protective immunity. New evidence points towards $C$. neoformans interaction with the cell-mediated immune system as being the basis by which $C$. neoformans infection is established. The conclusion that $C$. neoformans $\mathrm{H} 99-\gamma$ strain can induce primary immunity in mice and elicit a complete secondary protective immunity via induction of a CMI Th1 response may prove crucial for the future of cryptococcal research. Together with the discovery of cell surface mannoproteins, there exists exciting potential for human vaccine therapies that elicit a more appropriate immune response to $C$. neoformans infection. The development of a cryptococcal fungal vaccine will, undoubtedly, change the face of AIDS research and offer a much better chance of survival for immunocompromised patients and those undergoing immunosuppressive therapies.

\section{REFERENCES}

1. Bayer A, Choi C, Tillman D, et al. Fungal arthritis: V. Cryptococcal and Histoplasmal Arthritis. Semin Arth Rheum 1980; 9:218-227. 
2. Boden W, Fisher A, Medeiros A, et al. Bioprosthetic Endocarditis due to Cryptococcus Neoformans. J Cardiovasc Surg 1983; 24:164-166.

3. Chuck SL, Sande MA. Infections with Cryptococcus Neoformans in the Acquired Immunodeficiency Syndrome. N Engl J Med 1989; 321:794-9.

4. Kovacs, J. A., A. A. Kovacs, M. Polis, et al. Cryptococcosis in the Acquired Immunodeficiency Syndrome. Ann Intern Med 1985; 103:533-538.

5. Powderly, WG. Cryptococcal meningitis and AIDS. Clin Infect Dis 1993; 17:837-842.

6. Schutte C, Van der Meyden C, Magazi D. The impact of HIV on meningitis as seen at a South African Academic Hospital (1994 to 1998). Infection 2000: 28(1):3-7.

7. Currie BP, Casadevall A. Estimation of the prevalence of cryptococcal infection among HIV infected individuals in New York City. Clin Infect Dis 1994; 19:1029-1033.

8. Pappas PG, Perfect JR, Cloud GA, et al. Cryptococcosis in Human Immunodeficiency Virus-negative Patients in the Era of Effective Azole Therapy. Clin Infect Dis 2001; 33: 690-699.

9. Ecevit I, Clancy C, Schmalfuss I, et al. The Poor Prognosis of Central Nervous System Cryptococcosis among Nonimmunosuppressed Patients: a Call for Better Disease Recognition and Evaluation of Adjuncts to Antifungal Therapy. Clin Infect Dis 2006; 42: 1443-1447.

10. Aberg JA, Price RW, Heeren DM, Bredt B. A Pilot Study of the Discontinuation of Antifungal Therapy for Disseminated Cryptococcal Disease in the Patients with Acquired Immunodeficiency Syndrome, following Immunologic Response to Antiretroviral tTerapy. J Infect Dis 2002; 185:117982.

11. Shelburne SA, Darcourt III, White AC, Jr, et al. The Role of Immune Reconstitution Inflammatory Syndrome in AIDSRlated Cryptococcus Neoformans Disease in the Era of Highly Active Antiretroviral Therapy. Clin Infect Dis 2005; 40:10491052.

12. Kwon-Chung K, Varma A. Do Major Species Concepts Support One, Two or More Species within Cryptococcus Neoformans? FEMS Yeast Res 2006; 6:574-587.

13. Mitchell TG, Perfect JR. Cryptococcosis in the Era of AIDS-100 Years after the Discovery of C. neoformans. Clin Microbiol Rev 1995; 8:515-48.

14. Viviani M, Cogliati M, Esposto M, et al. Molecular analysis of 311 Cryptococcus Neoformans Isolates from a 30-month ECMM Survey of Cryptococcosis in Europe. FEMS Yeast Res 2006; 6(4):614-9.

15. Wang Y, Casadevall A. Susceptibility of Melanized and Nonmelanized Cryptococcus Neoformans to Nitrogen- and Oxygen-derived Oxidants. Infect Immun 1994; 62(7):3004-7.

16. Jacobson E, Tinnell S. Antioxidant Function of Fungal Melanin. J Bacteriol 1993; 175(21):7102-4.

17. Wang Y, Aisen P, Casadevall A. Cryptococcus Neoformans Melanin and Virulence: Mechanism of Action. Infect Immun 1995; 63(8):3131-6.

18. Jacobson E, Jenkins N, Todd J. Relationship between Superoxide Dismutase and Melanin in a Pathogenic Fungus. Infect Immun 1994; 62(9):4085-6.

19. Cox G, Harrison T, McDade H, et al. Superoxide Dismutase Influences the Virulence of Cryptococcus Neoformans by Affecting Growth within Macrophages. Infect Immun 2003; 71(1):173-80.

20. Perfect J, Wong B, Chang Y, et al. Cryptococcus Neoformans: Virulence and Host Defences. Med Mycol 1998; 36(1):79-86.

21. Wormley FL, Heinrich G, Miller J, et al. Identification and Characterization of an SKN7 Homologue in Cryptococcus Neoformans. Infect Immun 2005; 73(8):5022-30.

22. Kozel TR, Gulley WF, Cazin JJ. Immune Response to Cryptococcus Neoformans Soluble Polysaccharide: Immunological Unresponsiveness. Infect Immun 1977; 18:701-
707.

23. Sundstrom JB, Cherniak R. A Glucuronoxylomannan of Cryptococcus Neoformans Serotype A is a Type 2 T-cellindependent antigen. Infect Immun 1992; 60:4080-4087.

24. Murphy JW, Cozad GC. Immunological Unresponsiveness Induced by Cryptococcal Polysaccharide Assayed by the Hemolytic Plaque Technique. Infect Immun 1972; 5:896-901.

25. Goldman DL, Khine H, Abadi J, et al. Serologic Evidence for Cryptococcus Infection in the Early Childhood. Pediatrics 2001;107:E66

26. Abadi J, Pirofski L. Antibodies Reactive with the Cryptococcal Capsular Polysaccharide Glucuronoxylomannan are Present in Ssera from Children with and without HIV Infection. J Infect Dis 1999; 180:915-919.

27. Kuttin ES, Feldman M, Nyska A, et al. Cryptococcosis of the Nasopharynx in Mice and Rats. Mycopathologia 1988; 101:99104.

28. Kuhn, LR. Growth and Viability of Cryptococcus Hominis at Mouse and Rabbit Body Temperatures. Proc Soc Exp Biol Med 1939; 41:573-574.

29. Casadevall A and Perfect JR. In: editors name. Cryptococcus Neoformans. Washington, DC: ASM Press; 1998: 177-181.

30. Chen S. Sorrel T, Nimmo G, et al. Epidiology and Host- and Variety-dependent Characteristics of Infection due to Cryptococcus Neoformans in Australia and New Zealand. Australasian Cryptococcal Study Group. Clin Infect Dis 2000; 31:499-508.

31. Kozel, TR. Opsonization and Phygocytosis of Cryptococcus Neoformans. Arh Med Res 1993; 9:34-46.

32. Keller RG, Pfrommer GS, Kozel TR. Occurrences, Specificity, and Functions of Ubiquitous Antibodies in Human Serum that are Reactive with the Cryptococcus Neoformans Cell Wall. Infect Immun 1994; 62:215-220.

33. Houpt DC, Pfrommer GST, Young BJ, et al. Occurrences, Immunoglobulin Classes, and Biological Activities of Antibodies in Normal Human Sserum that are Reactive to Cryptococcus Neoformans Glucuronoxylomannan. Infect Immun 1994; 82:3857-3864.

34. Kozel TR, Wilson MA, Pfrommer GS, et al. Activation and Binding of Opsonic Fragments of C3 on Encapsulated and Nonencapsulated Cryptococcus Neoformans by Using an Alternative Complement Pathway Reconstituted from Six Isolated Patients. Infect Immun 1989; 57:1922-1927.

35. Miller MF, Mitchell TG. Killing of Cryptococcus Neoformans Strains by Human Neutrophils and Monocytes. Infect Immun 1991; 59:24-28.

36. Marr K, Jones G, Mody C. Contemplating the Murine Test Tube: Lessons from Natural Killer Cells and Cryptococcus Neoformans. FEMS Yeast Res 2006; 6(4):543-57.

37. Salkowski CA, Balish E. Role of Natural Killer Cells in Resistance to Systemic Cryptococcosis. J Leukocyte Biol 1991; 50:151-159.

38. Ganz T, Selsted ME, Szklarek D, et al. Defensins, Natural Peptide Antibiotics of the Human Neutrophils. J Clin Invest 1972; 76:1427-1435.

39. Diamond RD, Root RK, Bennett JE. Factors Influencing Killing of Cryptococcus Neoformans by Human Leukocytes in Vitro. J Infect Dis 1972; 125:367-376.

40. Hiemstra PS, Eisenhauer PB, Harwig LS, et al. Antimicrobial Proteins of the Murine Macrophages. Infect Immun 1993; 61:3038-3046.

41. Brummer E, Stevens DA. Anticryptococcal Activity of Macrophages: Role of Mouse Strain, C5, Contact, Phagocytosis an L-arginine. Cell Immunol 1994; 157:1-10.

42. Flesch IEA, Schwamberger G, Kaufman SHE. Fungicidal Activity of IFN-? Activated Macrophages. J Immunol 1989; 142:3219-3224.

43. Collins HL, Bancroft GJ. Cytokine Enhancement of 
Complement-dependent Phagocytosis by Macrophages: Synergy of Tumor Necrosis Factor-alpha and GranulocyteMacrophage Colony Stimulation Factor for Phagocytosis of Cryptococcus Neoformans. Eur J Immunol 1992; 22:1447-1454.

44. Diamond RD, Bennett JE. Growth of Cryptococcus Neoformans within Human Macrophages in Vitro. Infect Immun 1973; 7:231-236.

45. Feldmesser M, Kress Y, Novikoff $\mathrm{P}$ et al. Cryptococcus Neoformans is a Facultative Intracellular Pathogen in Murine Pulmonary Infection. Infect Immun 2000; 68:4225-4237.

46. Feldmesser M, Tucker S, and Casadevall A. Intracellular Parasitism of Mophages by Cryptococcus Neoformans. Trends Microbiol 2001; 6:273-8.

47. Vecchiarelli A. Immunoregulation by Capsular Components of Cryptococcus Neoformans. Med Mycol 2000; 38:407-417.

48. Devi SJN. Preclinical Efficacy of a GlucuronoxylomannanTetanus Toxoid Conjugate Vaccine of Cryptococcus Neoformans in a Murine Model. Vaccine 1996; 14:841-842.

49. Devi SJ, Schneerson R, Egan W, et al. Cryptococcus Neoformans Serotype A Glucuronoxylomannan-protein Conjugate Vaccines: Synthesis, Characterization, and Immunogenicity. Infect Immun. 1991; 59(10):3700-7.

50. Rivera J, Mukherjee J, Weiss LM, et al. Antibody Efficacy in Murine Pulmonary Cryptococcus Neoformans Infection: a Role for Nitric Oxide. J Immunol 2002; 168:3419-3427.

51. Williamson PR, Bennett JE, Polis MA, et al. Immunogenicity and Safety of a Conjugate Glucuronoxylomannan-tetanus Conjugate Vaccine in Volunteers. Clin Infect Dis 1993; 17:540.

52. Lim TS, Murphy JW. Transfer of Immunity to Cryptococcosis by T-enriched Splenic Lymphocytes from Cryptococcus Neoformans-sensitized Mice. Infect Immun 1980; 30:5-11.

53. Wormley FL, Perfect JR, Steele C, et al. Protection Against Cryptococcosis using Murine Interferon-gamma Producing Cryptococcus Neoformans Strain. Cryptococcus \& Cryptococcosis Tri-annual Meeting, June 2005.

54. Cauley LK, Murphy JW. Response of Congenitally Athymic (nude) and Phenotypically Normal Mice to Cryptococcus Neoformans Infection. Infect Immun 1979; 23:644-651.

55. Salkowski CA, Balish E. Susceptibility of Congenitally Immunodeficiant Mice to an Encapsulated Strain of Cryptococcus Neoformans. Can J Microbiol 1991; 37:834-839.

56. Salkowski CA, Balish E. Inflammatory Responses to Cryptococcosis in Congenitally Athymic Mice. J Leukocyte Biol 1991; 49:533-541.

57. Graybill JR, Ahrens J, Nealon T, Paque R. Pulmonary Cryptococcosis in the Rat. Am Rev Respir Dis 1983; 127:636640.

58. Wormley FL, Cox GM, Perfect JR. Evaluation of Host Immune Responses to Pulmonary Cryptococcosis using a TemperatureSensitive Cryptococcus Neoformans Calcineurin A Mutant Strain. Microb Pathog2005; 38:113-123.

59. Odom A, Muir S, Lim E, et al. Calcineurin is Required for Virulence of Cryptococcus Neoformans. EMBO J 1997; 16:2579-2589.

60. Hernandez Y, Arora S, Erb-Downward JR, et al. Distinct Roles for IL-4 and IL-10 in Regulating TH2 Immunity during Allergic Bronchopulmonary Mycosis. J Immunol 2005; 174(2):1027-36.

61. Furukawa K, Kobayashi M, Sasaki H, et al. Cryptococcal Encephalitis in Thermally Injured Mice is Accelerated by Type 2 T-cell Responses. Crit Care Med 2002; 30(7):1419-24.

62. Murphy JW, Schafer F, Casadevall A, et al. Antigen-induced Protective and Nonprotective Cell-mediated Immune Components against Cryptococcus Neoformans. Infect Immun 1998; 66:2632-2639.

63. Mandel MA, Grace GC, Orsborn KI, et al. The Cryptococcus Neoformans Gene DHA1 Encodes an Antigen that Elicits a Delayed-type Hpersensitivity Reaction in Immune Mice. Infect Immun 2000; 68:6196-6201.
64. Biondo C, Beninati C, Delfino D, et al. Identification and Cloning of a Cryptococcal Deacetylase that Produces Protective Immune Responses. Infect Immun 2002; 70(5):2383-91.

65. Levitz SM, Nong S, Mnasour MK, et al. Molecular Characterization of a Mannoprotien with Homology to Chitin Deacetylases that Stimulates T cell Responses to Cryptococcus Neoformans. Proc Natl Acad Sci USA 2001; 98:10422-10427.

66. Huang C, Nong SH, Mansour MK, et al. Purification and Characterization of a Second Immunoreactive Mannoproteins from Cryptococcus Neoformans that Stimulates T-cell Responses. Infect Immun 2002; 70:5485-5493.

67. Biondo C, Messina L, Bombaci M, et al. Characterization of Two Novel Cryptococcal Mannoproteins Recognized by Immune Sera. Infect Immun 2005; 73(11):7348-55.

68. Sallusto F, Cella M, Danieli C, et al. Dendritic Cells use Macropinocytosis and the Mannose Receptor to Concentrate Macromolecules in the Major Histocompatibility Complex Class II Compartment: Downregulation by Cytokines and Bacterial Products. J Exp Med 1995; 182:389-400.

69. Levitz SM, Specht CA. The Molecular Basis for the Immunogenicity of Cryptococcus Neoformans Mannoproteins. FEMS Yeast Res 2006; 6(4):513-24.

70. Pitzurra L, Cherniak R, Giammarioli M, et al. Early Induction of Interleukin-12 by Human Monocytes Exposed to Cryptococcus Neoformans Mannoproteins. Infect Immun 2000; 68(2):558-63.

71. Pietrella D, Cherniak R, Strappini C, et al. Role ofMannoprotein in Induction and Regulation of Immunity to Cryptococcus Neoformans. Infect Immun 2001; 69(5):2808-14.

72. Pietrella D, Mazzolla R, Lupo P, et al. Mannoprotein from Cryptococcus Neoformans Promotes T-helper Type 1 Anticandidal Responses in Mice. Infect Immun 2002; 70(12):6621-7.

73. Mansour MK, Schlesinger LS, Levitz SM. Optimal T cell Responses to Cryptococcus Neoformans Mannoproteins are Dependent on Recognition of Conjugated Carbohydrates by Mannose Receptors. J Immunol 2002; 168:2872-2879.

74. Vecchiarelli A, Retini C, Monari C, et al. Purified Capsular Polysaccharide of Cryptococcus Neoformans Induces Interleukin-10 Secretion by Human Monocytes. Infect Immun 1996; 64:2846-9.

75. Mansour MK, Yauch LE, Rottman JB, et al. Protective Efficacy of Antigenic Fractions in Mouse Models of Cryptococcosis. Infect Immun 2004; 72(3):1746-54.

Joel Schop has recently graduated with a B.S. in Biology from the University of Texas at San Antonio (UTSA) and is endeavoring to pursue medical school. He is currently studying under the supervision of Floyd Wormley Ph. D., microbiologist specializing in Cryptococcus neoformans, at the Margaret Batts Tobin Research Laboratory, UTSA. 